SUBJECT AREAS: APPLIED PHYSICS NANOFLUIDICS NEMS SENSORS

Received 22 January 2013 Accepted 2 May 2013

Published 20 May 2013

Correspondence and requests for materials should be addressed to M.T. (taniguti@sanken. osaka-u.ac.jp)

\section{Tracking single-particle dynamics via combined optical and electrical sensing}

\author{
Naoya Yukimoto', 2, Makusu Tsutsui' , Yuhui He' , Hirofumi Shintaku², Shoji Tanaka ${ }^{1,2}$, Satoyuki Kawano², \\ Tomoji Kawai' \& Masateru Taniguchi'
}

\begin{abstract}
'The Institute of Scientific and Industrial Research, Osaka University, 8-1 Mihogaoka, Ibaraki, Osaka 567-0047, Japan, ${ }^{2}$ Department of Mechanical Science and Bioengineering, Graduate School of Engineering Science, Osaka University, Toyonaka, Osaka 560-8531, Japan.
\end{abstract}

While fluorescent imaging has been extensively used for single-particle tracking, temporal and spatial resolution of the wide-field microscopy technology is not satisfactory for investigating fast-moving features. Here we report a method for probing nanometer-scale motion of an individual particle through a microstructured channel at sub-MHz by combining a resistive pulse technique to the optical sensing. We demonstrate unambiguous discriminations of translocation and non-translocation events inferred from spike-like electrical signals by fluorescence images captured synchronously to ionic current measurements. We also find a trajectory-dependent translocation dynamics of voltage-driven single-particles through a microchannel. This electrical/optical approach may find applications in sensor technologies based on micro- and nano-electromechanical systems.

ecent advance in nanotechnology has led to growing interest in replacing conventional optical approach of biosensing with electrical methods for improving the time and spatial resolution ${ }^{1-5}$. Nanopores and nanochannels are considered as one of the promising platforms with potential bioanalytical applications such as DNA sequencing and viral screening that can be utilized for real-time and label-free electrical single-molecule identifications ${ }^{4-8}$. The detection method probes a temporal change in the ionic current through a nanoscale conduit connecting the cis and trans chambers associated with an individual particle or molecule that partially excludes ions in there during translocation ${ }^{9}$. This volumetric effect enables sizing single-particles and investigating their fast translocation dynamics with sub-nanometer resolution ${ }^{10-12}$

Despite the unique and useful capability, however, the electrical approach lacks a way to ensure whether an analyte has been passed from cis to trans by the ionic current signatures alone as essentially any motions of substances nearby a nanopore would affect the ion pathways there and may cause resistive pulses that mimic translocations of target molecules ${ }^{13,14}$. In this sense, it is indispensable to combine additional functions for tracking the macroscopic flow of molecules to validate the envisaged translocation through a fluidic channel during single-molecule detections by the ionic current measurements ${ }^{15-17}$. In the present work, we have developed a microchannel sensor that allows incorporation of fluorescence microscopy observations to the electrical detections of single-particles by a resistive pulse method. Fluorescent imaging is a well-established technique for studying dynamics of single-molecules and -particles in micro- and nano-fluidics ${ }^{18-20}$. We exploited this to visualize particle motions under a hydrodynamic pressure and an electrophoretic field while monitoring the ionic current through a micro-fluidic channel (Fig. 1a). Although the time and spatial resolution of the optical observations is inadequate for resolving the fast kinetics of analytes electrophoretically passing through a microstructured conduit unless the channel length is made long enough to elongate the translocation duration for fluorescent detection $\mathrm{s}^{21}$, it offers vivid information for distinguishing whether a cis to trans migration has actually occurred at the moment when a spike-like signal in the cross-channel ionic current is detected (Fig. 1b), thereby enabling unambiguous electrical identification of single-particle translocation.

\section{Results}

Simultaneous measurements of cross-channel ionic current $I$ and fluorescence imaging were performed using a microstructured fluidic channel lithographically-defined in a $150 \mu \mathrm{m}$ thick cover glass substrate under a dc voltage $V_{\mathrm{b}}$ (Fig. 1c-g). The pressure difference between cis and trans was minimized by adjusting the height of syringes connected to the channel. The microchannel had a bow-tie shape with height $h=1.2 \mu \mathrm{m}$, length $L=$ $4.0 \mu \mathrm{m}$, and width $w_{1}=2.6 \mu \mathrm{m}$ and $w_{2}=1.4 \mu \mathrm{m}$ at the orifice and the necked part, respectively (Supplementary 
a

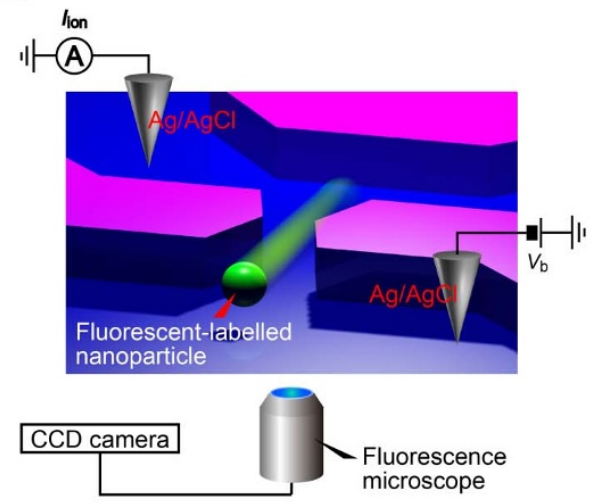

b

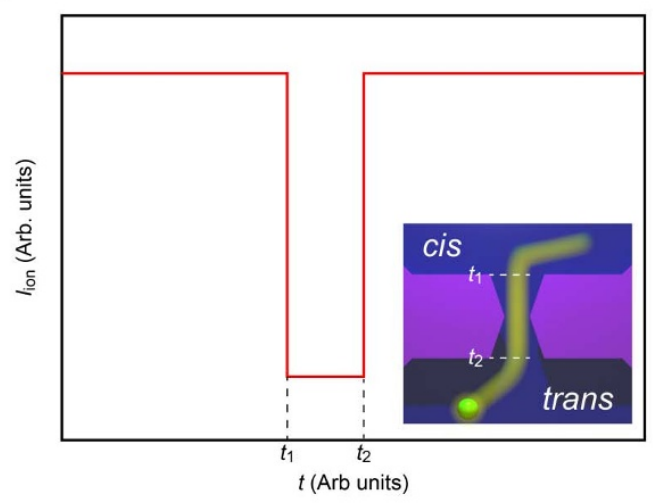

C

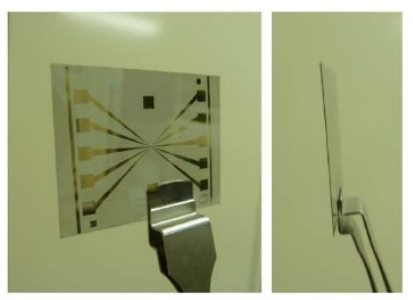

d

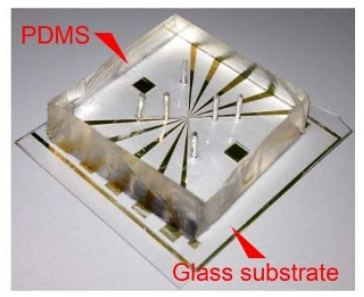

e

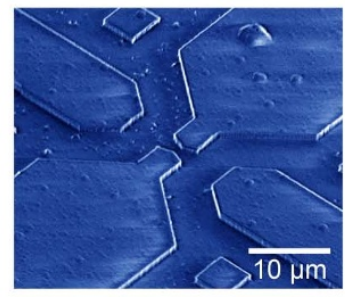

f

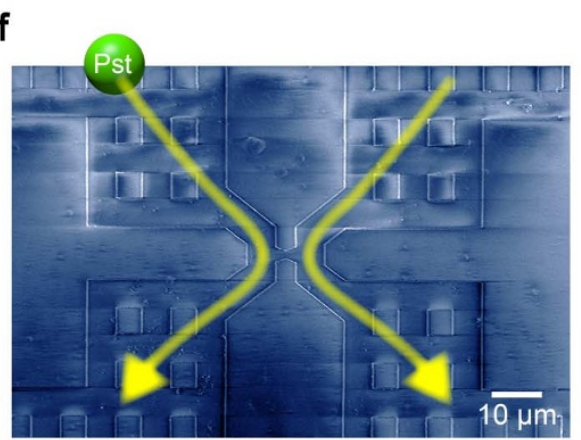

g

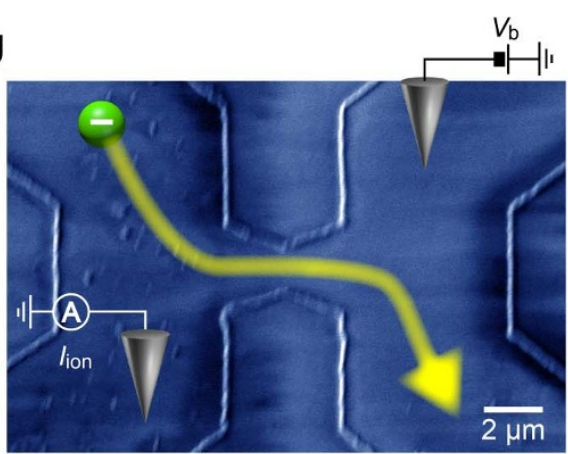

Figure 1 Fluorescence microscope setup used for simultaneous optical and electrical sensing of micro-particles. (a), A sketch of the experimental setup. (b), fluorescently-dyed polystyrene (Pst) beads of diameter $1 \mu \mathrm{m}$ are voltage-driven to pass through a microchannel fabricated on a cover glass using two $\mathrm{Ag} / \mathrm{AgCl}$ electrodes. Meanwhile, fluorescence images are taken from back of the substrate concurrently with measurements of the ionic current through the channel. (b), A conceptual result of the optical/electrical detections. Translocation of an individual particle through a microchannel yields a resistive pulse in an $I-t$ trace. The corresponding fluorescence images reveal trajectories of the particle flow. $t_{1}$ and $t_{2}$ denote the time when a particle enters and exits the channel. (c-d), Overview of the fluidic channel sample. The substrate is a standard cover glass. The electrode pattern was utilized as external markers for delineating microchannels (c). A PDMS block was adhered on the substrate for sealing the channel (d). (e-g), False-colored scanning electron microscopy (SEM) images of the microchannel. It consists of a bow-tie shaped conduit connecting the two reservoir channels filled with micropillar arrays (e). Pst beads are injected into the channels by a hydrodynamic pressure (f). Occasionally, the negatively-charged particles electrophoretically translocate through the microchannel by the strong electric field $(\mathrm{g})$.

Fig. S1). A voltage of $V_{\mathrm{b}}=10 \mathrm{~V}$ was applied to the channel, which builds an electric field for assisting electrophoretic translocation of a fluorescently-dyed polystyrene (Pst) beads of a nominal diameter of $1.1 \mu \mathrm{m}$ containing carboxylate groups (TransFluoSpheres ${ }^{\circledR}$ beads) through the microchannel by exerting an electrostatic force for overcoming an energy barrier associated with electrostatic interactions (Fig. 1g) ${ }^{22}$. The acquired $I-t$ curve showed open channel current $I_{\text {open }}$ of about $69 \mathrm{nA}$ (Fig. 2a). This fairly agrees with analytical estimation of $I_{\text {open }}=V_{\mathrm{b}} /\left(R_{\text {micro }}+R_{\text {ext }}\right)=69.6 \mathrm{nA}$. In the equation, $R_{\text {micro }}=$ 17.2 $\mathrm{M} \Omega$ is the resistance in the bow-tie shaped microchannel assuming the resistivity of TE buffer $\rho=10 \Omega \mathrm{m}$ and $R_{\text {ext }}=$ 126.4 $\mathrm{M} \Omega$ is the resistance in the large channel with micro-pillar arrays (details are described in Supporting Information) ${ }^{23}$.

Transient decrease in $I$ was observed in stochastic time intervals (Fig. 2a), which is usually interpreted as denoting independent translocation events of individual particles through the microchannel ${ }^{22,24,25}$.
Here, we focus on the four resistive pulses labeled as $A$ through $D$ and verify whether Pst single-particles have actually been transported from cis to trans at the moment when the ionic current signatures are detected by the fluorescence images recorded during the course of I measurements (the whole motion picture can be found in Movie 1). It is noticeable that after the event $A$, the background current decreased by about $1 \mathrm{nA}$ from $I_{\text {open }}=69 \mathrm{nA}$ and stayed at that current level until the subsequent signal $B$ appeared. Although this anomalous feature infers particle translocations through the microchannel, the electrical signatures alone cannot provide clear evidence. In fact, the series of $I$-spikes can be attributed to either translocation of one particle involving temporal trap at the orifice via adhesion on the wall surface ${ }^{23}(A)$ and detrapping several seconds later $(B)$ or two individual particles sequentially passing through the channel.

On the other hand, concurrent optical imaging of Pst particles provide useful information for addressing the uncertainty in the 
a

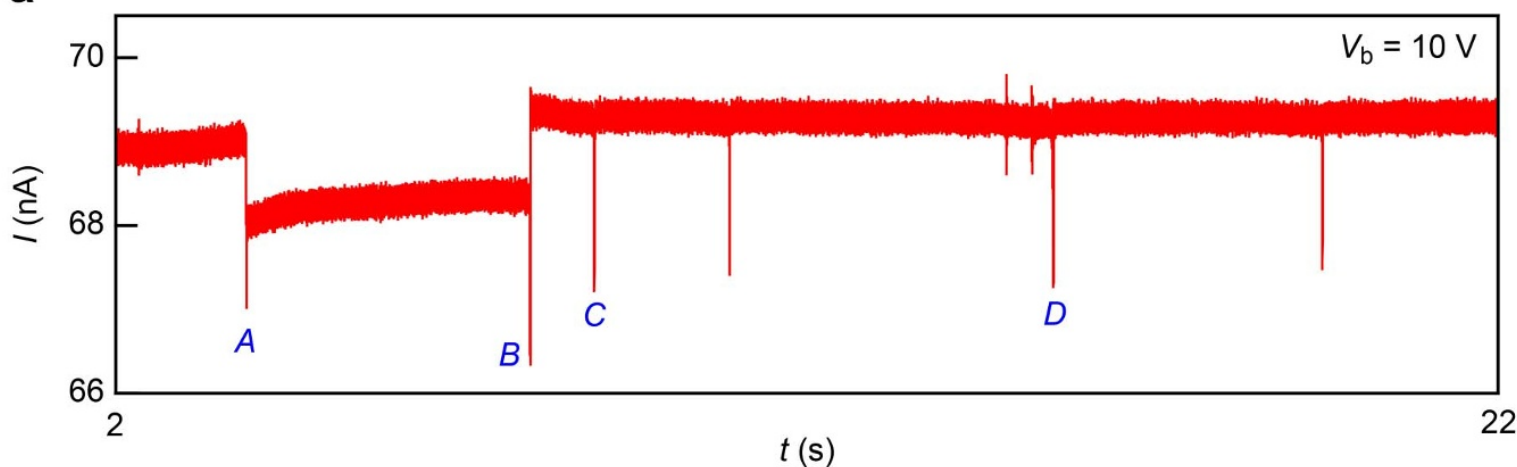

b

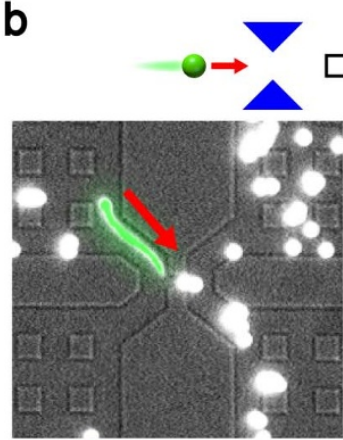

(A) $t=3.53$
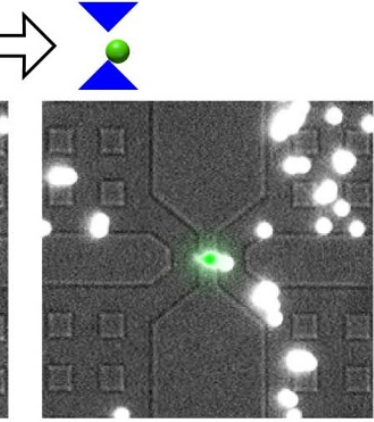

$t=3.57$

C

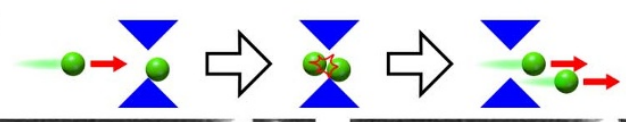

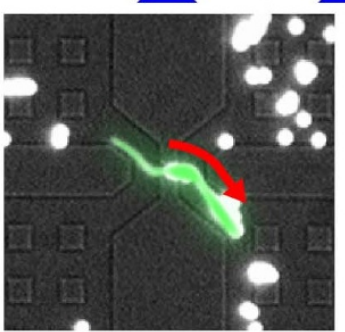

(B) $t=7.67$

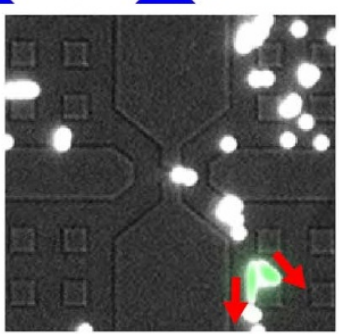

$t=7.73$

d

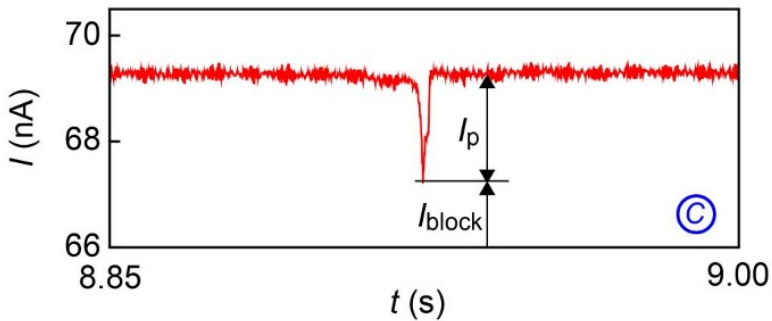

e

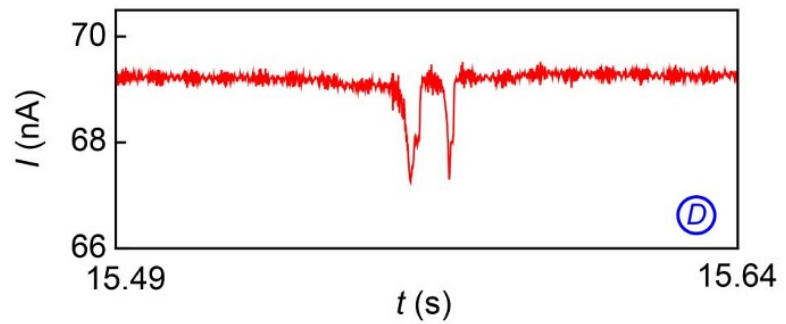

g

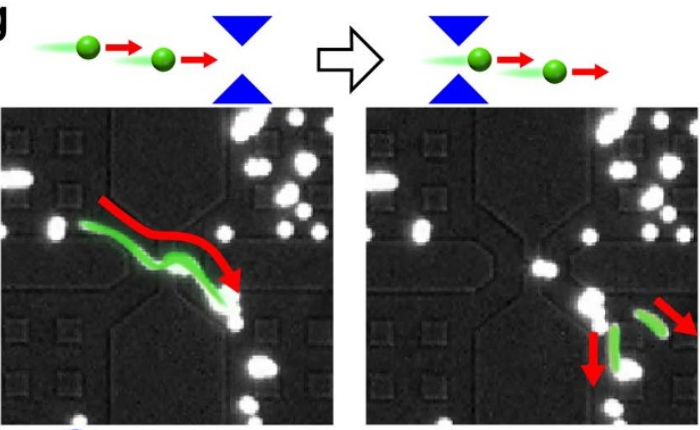

(D) $t=15.56$

f
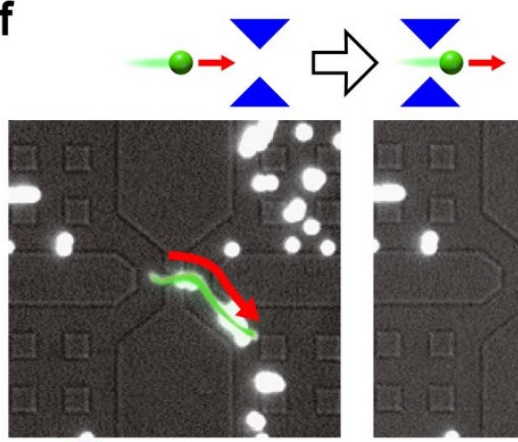

(C) $t=8.92$

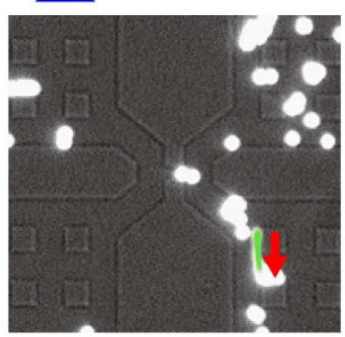

$t=8.97$

Figure $2 \mid$ Profiling of single-particle translocation events by an optical/electrical combined analysis. (a), Temporal change in the ionic current through a bow-tie microchannel $I$ recorded under a voltage $V_{\mathrm{b}}=10 \mathrm{~V}$. Pst particles were flowed through one of the reservoir channels by a hydro-pressure. Four characteristic spike signals are marked $A$ through $D$. Data are compressed by 100 points for clarity. (b-c), Consecutive snapshots showing particle trapping in the microchannel during signal $A(\mathrm{~b})$ and subsequent detrapping induced by electrophoretic translocation of another Pst bead that collide with the trapped particle at the moment of signal $B$ (c). Schematic illustrations of the translocation mechanism are displayed above the images. Moving particles are colored green. (d-e), Magnified views of $C(\mathrm{~d})$ and $D(\mathrm{~d}) . I_{\mathrm{block}}$ and $I_{\mathrm{p}}$ denote the minimum $I$ in the current spike and the blockage current, respectively. ( $\mathrm{f}-\mathrm{g})$, Fluorescence images recorded at signal $C(\mathrm{f})$, and $\mathrm{D}(\mathrm{g})$. Schematics of the translocation processes are shown above the micrographs. These particle motions are displayed more clearly in the video image Movie 2 of fluorescence observations. 
electrical detections ${ }^{16,26}$. Consecutive snapshots recorded at $30 \mathrm{~Hz}$ (Fig. 2b) highlight a movement of single-particle into the microchannel at the point when signal $A$ occurs (Bright dots at fixed positions are immobilized Pst particles by strong non-specific adhesion to the $\mathrm{SiO}_{2}$ channel). This suggests partial blocking of the conduit by the micro-bead adhered on an adjacent wall surface that diminishes ion pathways through the channel and cause the decrease in $I_{\text {open }}$ seen in Fig. $2 \mathrm{a}^{23}$. Subsequently, fluorescence images elucidate another Pst bead propelled through the microchannel when signal $B$ is detected. What is interesting here is that the particle virtually splits into two after the translocation (Fig. 2c). Moreover, the background ionic current returns to $I_{\text {open }}$ following the event $B$. The concomitant recover of $I$ indicates release of the trapped Pst via collision with another particle entering the channel as depicted schematically in Fig. 2c. The detrapped particle would then be repelled from the microchannel by electrophoresis along with the second bead, which causes the apparent splitting in the optical micrographs. Similarly, magnified views of signals $C$ and $D$ render single and double spikes, respectively (Fig. 2d-e). The height of spike $I_{\mathrm{p}}$ is $1.89 \mathrm{nA}$, which is in accordance with the theoretical $I_{\mathrm{p}}=1.83 \mathrm{nA}$ assuming a $1.1 \mu \mathrm{m}$ diameter sphere trapped in the narrowest constriction of the bowtie type conduit (see Supporting Information for details). Therefore, these resistive pulses are naturally ascribed to single-particles passing through the microchannel ${ }^{22,24,25}$. Meanwhile, the corresponding fluorescence images indeed clearly capture the translocation dynamics. For signal $C$, optical observations reveal a motion of a dot moving through the microchannel (Fig. 2f). On the other hand, the micrographs for signal $D$ detect two dots moving toward the trans (Fig. 2g), which corroborates two individual particles passing through the channel during signal $D$ wherein double-spikes are detected (Fig. 2e). These results demonstrate the unique ability of fluorescence detections that enable profiling of complicated single-particle translocation dynamics involving particle-particle interactions in a microfluidic channel when utilized with the ionic current trace analysis.

The optical/electrical sensing was applied here to evaluate the translocation dynamics of single-particles in a fluidic channel. We employed a linear microchannel with width, length, and height of $1.4 \mu \mathrm{m}, 4.0 \mu \mathrm{m}$, and $1.2 \mu \mathrm{m}$, respectively (Fig. $3 \mathrm{a}$; see also Supplementary Fig. S4). Current spikes were detected in $I-t$ curves measured under $V_{\mathrm{b}}=5 \mathrm{~V}$ (Fig. 3b), which were characterized by the height $I_{\mathrm{p}}$ and the width $t_{\mathrm{d}}$ (Fig. 3c). Concurrent fluorescence imaging identifies particle-by-particle translocations through a microchannel synchronous to the occurrence of resistive pulses (Fig. 3d; a video image is also available in Supporting Information Movie 2).

Statistically, 233 out of 265 spikes are in fact originated from ionic current blockade via particle translocations (see Supplementary a

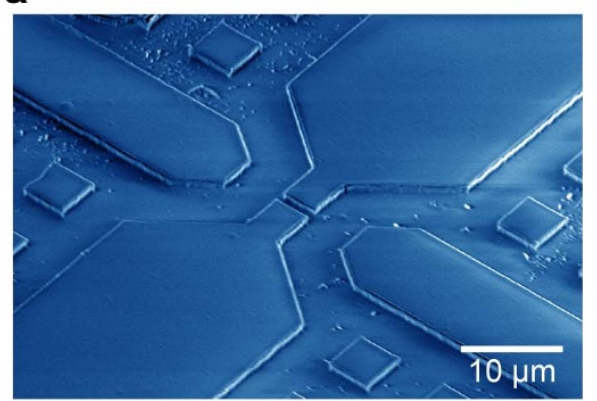

C

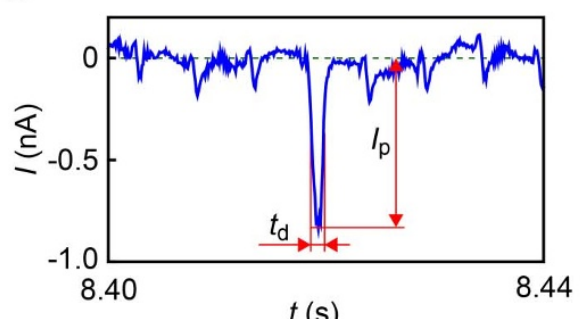

b

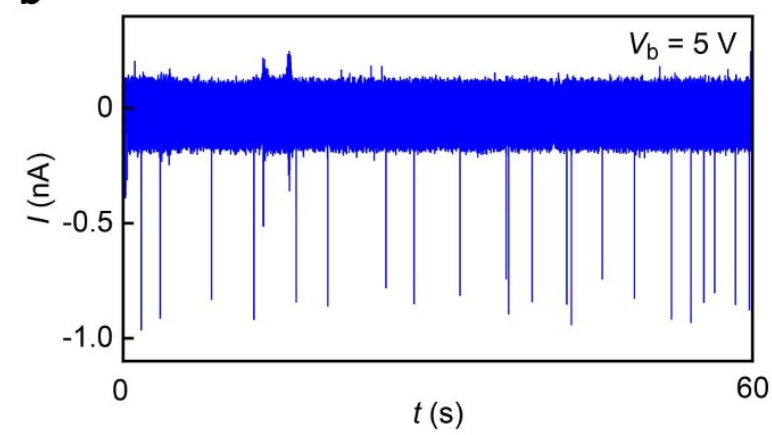

d

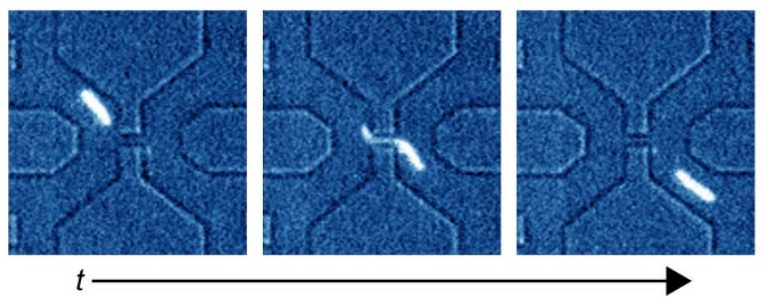

g
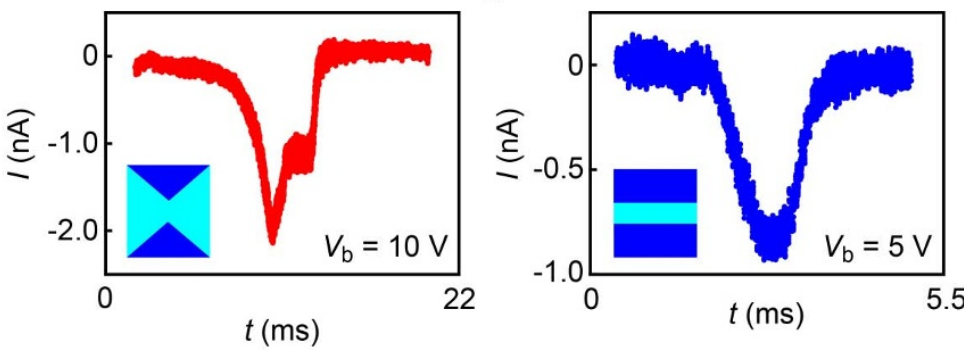

Figure 3 Statistical characterization of particle sizes by optical/electrical measurements. (a), SEM micrograph of a fluidic channel with a linear configuration used for the single-particle detections. (b), I- $t$ curve obtained under $V_{\mathrm{b}}=5 \mathrm{~V}$ with $1 \mu \mathrm{m}$ Pst beads introduced into one side of the microchannel. Background current is offset to zero. Data are compressed to $10 \mathrm{kHz}$ for clarity. (c), Close view of a current spike in (b). $I_{\mathrm{p}}$ and $t_{\mathrm{d}}$ are the height and the width of resistive pulses. (d), Optical imaging during the signal shown in (c). A particle is moving through the channel (from left to right). (e), Distributions of $I_{\mathrm{p}}$. Red curve is a Gaussian fit to the histogram. ( $\left.\mathrm{f}-\mathrm{g}\right)$, Resistive pulses detected when a $1.1 \mu \mathrm{m}$ polystyrene particle passing through the bow-tie (f) and the linear channel $(\mathrm{g})$ as confirmed by the fluorescent imaging. Insets are the schematic top view. 
a

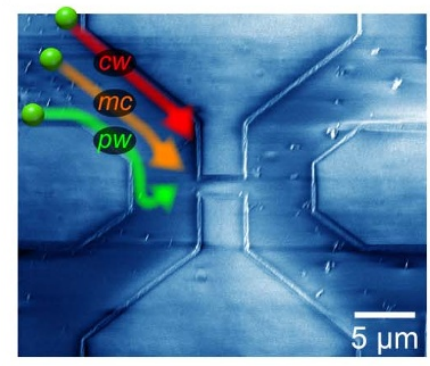

b

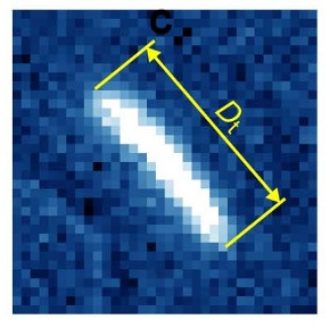

e

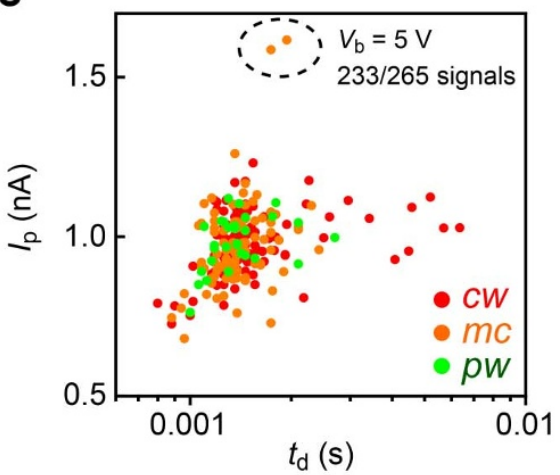

h

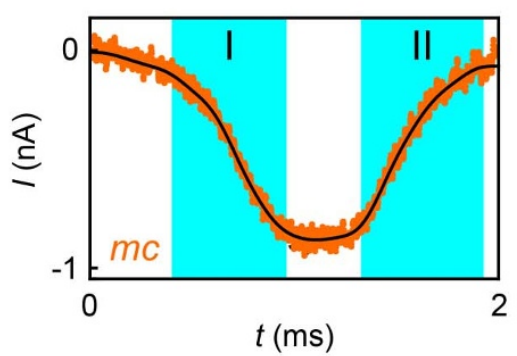

C
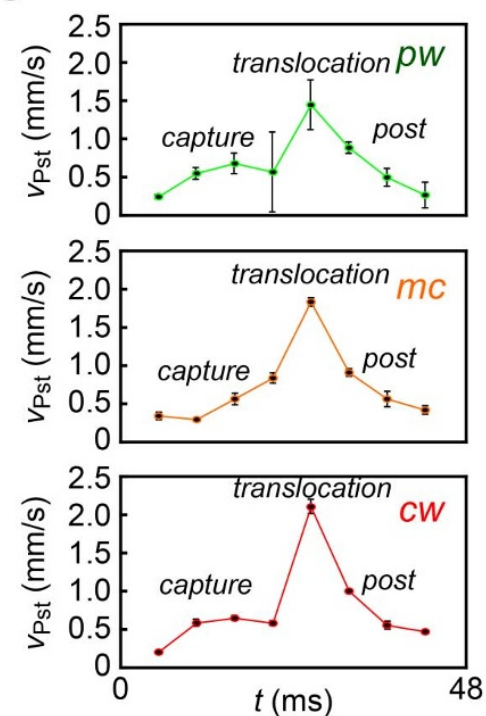

f

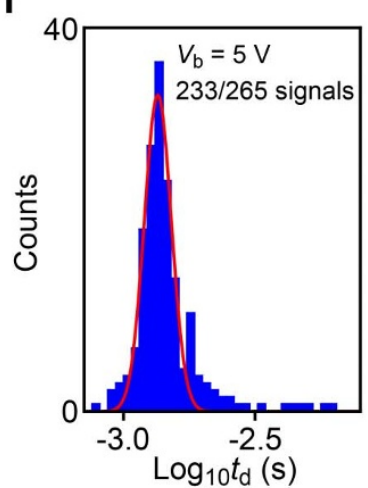

i

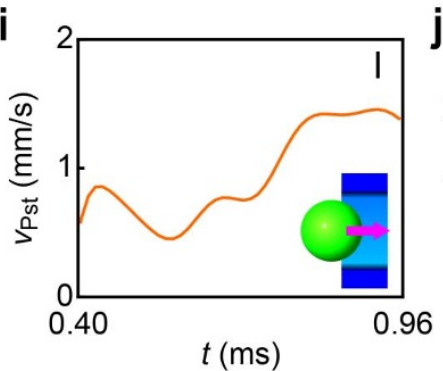

d
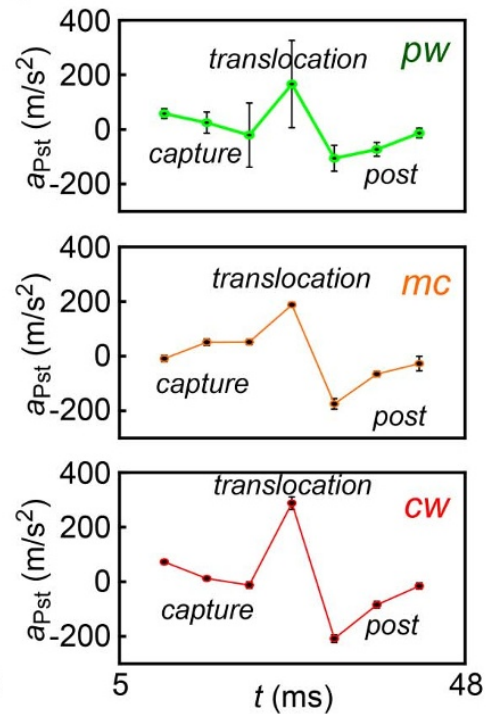

g

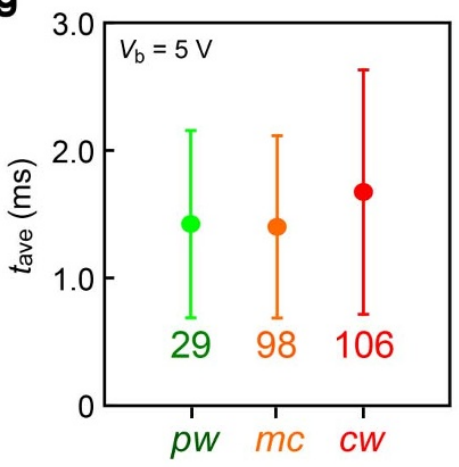

Figure $4 \mid$ Combined analysis of single-particle translocation dynamics. (a), Schematic explanation of three distinct particle trajectories revealed by fluorescent imaging. $p w$ and $c w$ stand for the particle flow along the side walls of the micropillar and the channel, respectively. $m c$ denotes the particle pathway in the middle of the channel. (b), Fluorescence image of a $1.1 \mu \mathrm{m}$ polystyrene (Pst) particle moving through the fluidic channel. $D_{\mathrm{t}}$ corresponds to the distance the particle has travelled within the exposure time of approximately $5 \mathrm{~ms}$. (c-d), Velocity $v_{\mathrm{Pst}}(\mathrm{c})$ and acceleration $a_{\mathrm{Pst}}(\mathrm{d})$ of the Pst particles obtained from $D_{\mathrm{t}}$. Each data are an average of three events. Color coding is the same as in (a). (e), Scatter plots of the width $t_{\mathrm{d}}$ and the height $I_{\mathrm{p}}$ of the resistive pulses at $V_{\mathrm{b}}=5 \mathrm{~V}$. Only signals that have been confirmed to be caused by translocation of single-particles by the simultaneous fluorescent imaging were used (233 out of 265 signals). Color coding distinguishes the particle trajectories. Two plots circled by a dotted line are attributed to translocation of two-particle-clusters. (f), Distribution of $\log _{10} t_{\mathrm{d}}$ constructed with the 233 current spikes. Red curve is Gaussian fitting to the histogram. $(\mathrm{g})$, Average of translocation duration $t_{\mathrm{ave}}$ for the three distinct single-particle trajectories at $V_{\mathrm{b}}=5 \mathrm{~V}$. The number of resistive pulses used is 29,98 , and 106 for $p w, m c$, and $c w$ processes as indicated in the figure. Error bars show the standard deviation. (h-j), $v_{\text {Pst }}$ in the microchannel assessed from the resistive pulse (h) during the particle entrance (i) and exit (j).

Fig. S5 for examples of $I$ signals assigned as non-translocation events). A histogram of $I_{\mathrm{p}}$ revealed a narrow distribution indicating monodispersed Pst beads in a TE buffer (Fig. 3e). The peak is located at $I_{\mathrm{p}}=0.98 \mathrm{nA}$, which is close to the analytical estimation of $I_{\mathrm{p}}=$ $1.11 \mathrm{nA}$ (see Supporting Information). Furthermore, the full-width at half-maximum is $0.23 \mathrm{nA}$. This is ascribed to a $4 \%$ variation of Pst particle sizes, or more specifically $d_{\mathrm{Pst}}=1.10 \mu \mathrm{m} \pm 40 \mathrm{~nm}$, which is within the nominal size distributions of TransFluoSpheres ${ }^{\circledR}$ beads ranging from 1 to $5 \%$.

There is an obvious difference in the line shapes of the ionic current signatures acquired in the bow-tie (Fig. 3f) and the linear channels (Fig. 3g). Recent nanopore experiments ${ }^{27}$ report that the 
I-profiles during single-particle translocations reflect the fine topology inside the conduit; more (less) ions are blocked when a microbead flow through a locally narrowed (widened) region in a pore $^{27}$. The differing $I$-waveforms in Figs. $3 \mathrm{f}-\mathrm{g}$ can be explained in a similar way: a particle moving through the sharp-edged constriction of the bow-tie geometry yields the V-shaped spikes whereas that moving in the linear conduit causes a constant $I_{\text {block }}$ for longer distance $(4 \mu \mathrm{m})$ resulting in the U-shaped waveform (the step in Fig. $3 \mathrm{f}$ indicate an existence of a trap site in the microchannel).

Fluorescence imaging allows a classification of single-particle translocations into three processes in the capture stage: a bead moving through the middle $(m c)$ or along the side surfaces of either the channel $(c w)$ or the pillar $(p w)$ as described in Fig. 4a (fluorescence images showing the three processes can be found in Supplementary Fig. S6). To shed light on the particle dynamics, we extracted the speed of single-particles $v_{\text {Pst }}$ from the trajectory length $D_{\mathrm{t}}$ in each optical micrograph taken with the exposure time of about $5 \mathrm{~ms}$ (Fig. 4b). Plots of $v_{\text {Pst }}$ (Fig. 4c) and the acceleration $a_{\text {Pst }}$ (Fig. 4d) with respect to time demonstrate sharp rises during field-driven translocations through the microchannel and subsequent deceleration by hydrodynamic dragging in trans. An apparent difference among the three mechanisms can be found in a capture phase; whereas $m c$ particles are being accelerated, those move along the side walls ( $c w$ and $p w$ ) are experiencing a retarding force most ascribable to particle-wall interactions as marked by the steady decrease in $a_{\mathrm{Pst}}$ (Figs. S7-8).

\section{Discussion}

The advantage of the simultaneous detection approach is exploited here to explore influence of a particle history in the pre-translocation stage on single-particle dynamics in a microchannel. The in-channel average velocity $v_{\mathrm{Pst}}$ of Pst beads can be assessed from the width of resistive pulses that represents the time-of-flight of single-particles through the $4 \mu \mathrm{m}$-long conduit (Fig. $3 \mathrm{c})^{28} . I_{\mathrm{p}}$ versus $t_{\mathrm{d}}$ scatter plots were generated from the $233 \mathrm{I}$ spikes and classified into the three groups (Fig. 4e). The two data points with anomalously large $I_{\mathrm{p}}$ of about $1.6 \mathrm{nA}$ is assigned to translocation of a two-particle-cluster (Supplementary Fig. S9). We find no conspicuous dependence of $I_{\mathrm{p}}$ on the particle trajectories at the capture stage. In contrast, $t_{\mathrm{d}}$ of $\mathrm{cw}$ (red plots) extends to much longer time scales than the other two, suggesting trajectory-dependent particle kinetics in the microchannel.

More quantitatively, $t_{\mathrm{d}}$ distribution of the whole events shows a monomodal profile whose peak is approximately at $t_{\mathrm{d}}=1.3 \mathrm{~ms}$ (Fig. 4f). The average particle speed is extracted from this as $v_{\mathrm{Pst}}=$ $L / t_{\mathrm{d}}=3.1 \mathrm{~mm} / \mathrm{s}$. The fact that $v_{\mathrm{Pst}}$ is underestimated in fluorescence observations is a consequence of the low temporal resolution: a particle passes though the microchannel within the exposure time of approximately $5 \mathrm{~ms}$ for recording an image with the optical microscope (Supplementary Fig. S6). When dividing $t_{\mathrm{d}}$ data into the three groups, the average trapping duration $t_{\mathrm{ave}}$ for $p w$ and $m c$ is estimated as $1.4 \pm 0.7 \mathrm{~ms}$ whereas that of $c w$ is $1.7 \pm 0.9 \mathrm{~ms}$ (Fig. $4 \mathrm{~g}$ ). The longer $t_{\text {ave }}$ for $c w$ by $21 \%$ suggests more significant retarding of the electrophoretic mobility via direct interactions of Pst particles with channel side walls ${ }^{10}$ and electroosmotic dragging there in direction opposite to the translocation for the native negative surface charges of the $\mathrm{SiO}_{2}$ channel ${ }^{29}$ evident from fluorescent observations showing the beads travel along the wall throughout the capture and translocation stages, unlike $p w$ and $m c$ processes where particles enter the microchannel without touching channel walls ${ }^{30}$. This manifests the importance of regulating the pre-translocation aspects of particle motions for achieving fine-control of the dynamics in microstructured fluidic channels.

The resistive pulses enable velocity profiling of the fast-moving singe-particles in the microchannel. We estimated the position of a particle $x$ along the $4 \mu \mathrm{m}$-long channel from the entrance and exit effects on the ionic transport (Supplementary Fig. S10). The velocity is then calculated numerically from $x-t$ plots as $v_{\mathrm{Pst}}=\Delta x / \Delta t$ (Fig. $4 \mathrm{~h}$ ). We find a rapid increase in $v_{\text {Pst }}$ when a particle entering the channel (Fig. 4i), which reflects acceleration via a huge local electric field at the channel. In contrast, the electrophoretic migration is significantly retarded upon escaping (Fig. 4j) by hydrodynamic dragging under a weak exterior electric field. The series of motion is in qualitative agreement with a simulation of voltage-driven particle translocation through a fluidic channel ${ }^{31}$.

The above results serve to prove that the electrical/optical combined analysis will be a powerful tool for real-time tracking of singleparticles over an extensive spatial and dynamic range: optical sensing capture the overall particle trajectories that complement the electrical detections for velocimetry of fast moving features at nanoscale. In addition, the capability of ensuring the presence and absence of single-particles or molecules in a specified area at the moment when an electrical signal is detected will be most useful in validating the electrical signatures of uncertain origin, such as ionic current blockage during single-particle translocation through a low-aspectratio conduit ${ }^{23,32}$ and transverse tunneling current through singlemolecules trapped in an electrode-embedded nanochannel ${ }^{33,34}$.

\section{Methods}

Fabrication of microfluidic channels. We first coated a $1.5 \mu \mathrm{m}$ thick $\mathrm{SiO}_{2}$ layer on a standard cover glass substrate of thickness about $150 \mu \mathrm{m}$ by chemical vapor deposition. We then patterned microelectrodes by a photo-lithography process with a photo-resist AZ5206E. After that, we deposited $20 \mathrm{~nm}$ thick Au with a Pt adhesion layer of thickness $2 \mathrm{~nm}$ using a radio-frequency (RF) magnetron sputtering method. Subsequently, the substrate was immersed in N,N-dimethylformamide for half a day and ultrasonicated for lift-off. The thus formed micro-pattern was used as external markers in the subsequent electron-beam (EB) lithography process. In prior to that, we coated the substrate with a $150 \mathrm{~nm}$ thick $\mathrm{Cr}$ by the RF sputtering. Then, we delineated a fluidic channel using $30 \mathrm{keV}$ electron beam with a resist ZEP520A-7, followed by removal of the exposed $\mathrm{Cr}$ layer by wet etching. The remaining $\mathrm{Cr}$ served as an etching mask during isotropic reactive ion etching $\left(\mathrm{CF}_{4}, 100 \mathrm{~W}\right)$ of $\mathrm{SiO}_{2}$. As a result, we obtained two reservoir channels of uniform height $1.2 \mu \mathrm{m}$ with average width of about $5.0 \mu \mathrm{m}$. The particle sensing micro-channel connecting the reservoirs had a bow-tie shape $2.6 \mu \mathrm{m}$ wide at the orifices and the narrowest constriction of width $1.2 \mu \mathrm{m}$. We prepared square micro-pillars for preventing roof collapse after sealing of the channel at the top with a polymer block made of polydimethylsiloxane (PDMS).

PDMS sealing. The microchannel device was sealed with a PDMS block. We formed four fluidic channels on one side of the polymer block by curing PDMS (Sylgard 184) at 70 degree Celsius for 1 hour on an SU-8 mold fabricated on a silicon wafer using a photo-lithography process. These four channels were aligned to the microfluidic channels using a stereomicroscope upon sealing. In prior to this, both the PDMS block and the device substrate were exposed to oxygen plasma (50 W for 10 seconds) for strengthening $\mathrm{SiO}_{2}$-PDMS bonding. The two surfaces were then immediately bonded together. Two pairs of inlet and outlet ports were created for controlling the particle flow via the external hydraulic pressure. Additional two holes punched in the PDMS were used to insert $\mathrm{Pt}$ rods of diameter $0.8 \mathrm{~mm}$ coated with $\mathrm{Ag} / \mathrm{AgCl}$ paste (BAS) for the ionic current measurements through the microchannel.

Fluorescent imaging. The microfluidic channel device was placed in a home-built faraday cage equipped with a fluorescence microscope (Olympus IX71) and an electron multiplying charge coupled device (EMCCD) camera (Andor Ixon Ultra). A TE buffer ( $10 \mathrm{mM}$ tris-HCl, $1 \mathrm{mM}$ EDTA) solution containing fluorescent-labelled polystyrene (Pst) particles of nominal diameter $1.1 \mu \mathrm{m}$ (TransFluoSpheres ${ }^{\circledR}$ beads) was introduced into one side of the microfluidic channels from an inlet port while the other side was filled with TE buffer. The liquid flowed toward the outlet by capillary force without any pressure. We irradiated ultra-violet (UV) light from the top for excitation of fluorophores decorated on the Pst particles. Fluorescence images were acquired using a suitable filter set from the back side of the thin glass substrate under $60 \times$ magnification by the EMCCD camera at a frame rate of $30 \mathrm{~Hz}$ or $189 \mathrm{~Hz}$ and recorded in a RAID storage media.

Ionic current measurements. A constant dc voltage $V_{\mathrm{b}}$ was applied to the microchannel through one of the $\mathrm{Ag} / \mathrm{AgCl}$ electrodes using a picoammeter source (Keithley 6487). The other one is connected to a current amplifier (Axopatch 200B), which amplified the current through the channel with a $50 \mathrm{M} \Omega$ feedback resistor $(\beta=$ 0.1 ). Output voltage was low-pass filtered at $100 \mathrm{kHz}$, digitized at $1 \mathrm{MHz}$ (NI PXI5922), and stored into RAID (NI HDD-8265).

Regulation of hydrodynamic flow. We first examined regulation of the hydrodynamic flow in the channel by the hydraulic head pressure through manipulating the height of syringes connected to the device. The Pst particles dispersed in a TE buffer at a concentration $0.002 \%$ solid was injected into a fluidic 
channel through holes punched in a PDMS block (Fig. S3). The ultra-violet (UV) excited fluorophores illuminate Pst particles imaged as blight spots through an objective lens from back of the glass substrate. Fluorescence microscopy images showed trajectories of Pst particles moving rapidly through a U-shaped channel under a high hydrodynamic pressure with $V_{\mathrm{b}}=0 \mathrm{~V}$ (Movie 1). None of the particles flowed into the micro-channel even when a pressure difference was built between $c$ is and trans, suggesting an energy barrier associated with electroosmotic flow at the small orifice of the microchannel that hinders translocation of $1.1 \mu \mathrm{m}$-sized Pst spheres.

1. Roy, S. \& Gao, Z. Nanostructure-based electrical biosensors. Nano Today 4, 318-334 (2009).

2. Chen, X. et al. Electrical nanogap devices for biosensing. Mat. Today 13, 28-41 (2010).

3. Robinson, K. Semiconductors charge into sequencing. Nat. Biotechnol. 29, 805-807 (2009).

4. Branton, D. et al. The potential and challenges of nanopore sequencing. Nat. Biotechnol. 26, 1146-1153 (2008).

5. Zwolak, M. \& Di Ventra, M. Colloquim: Physical approaches to DNA sequencing and detection. Rev. Mod. Phys. 80, 141-165 (2008).

6. Howorka, S. \& Siwy, Z. Nanopore analysis: sensing of single molecules. Chem. Soc. Rev. 38, 2360-2380 (2009).

7. Venkatesan, B. M. \& Bashir, R. Nanopore sensors for nucleic acid analysis. Nat. Nanotechnol. 6, 615-624 (2011).

8. Wanunu, M. Nanopores: A journey towards DNA sequencing. Phys. Life Rev. 9, 125-158 (2012).

9. DeBlois, R. W. \& Bean, C. P. Counting and sizing of submicron particles by the resistive pulse technique. Rev. Sci. Instrum. 41, 909-915 (1970).

10. Garaj, S. et al. Graphene as a subnanometre trans-electrode membrane. Nature 467, 190-193 (2010)

11. Rosenstein, J. K., Wanunu, M., Merchant, C. A., Drndic, M. \& Shepard, K. L. Integrated nanopore sensing platform with sub-microsecond temporal resolution. Nat. Methods 9, 487-492 (2012).

12. Manrao, E. A. et al. Reading DNA at single-nucleotide resolution with a mutant MspA nanopore and phi29 DNA polymerase. Nat. Biotechnol. 30, 349-353 (2012).

13. van den Hout, M., Kruddle, V., Janssen, X. J. A. \& Dekker, N. H. Distinguishable populations report on the interactions of single DNA molecules with solid-state nanopores. Biophys. J. 99, 3840-3848 (2010).

14. Vlassarev, D. M. \& Golovchenko, J. Trapping DNA near a solid-state nanopore. Biophys. J. 103, 352-356 (2012).

15. Ando, G., Hyun, C., Li, J. \& Mitsui, T. Directly observing the motion of DNA molecules near solid-state nanopores. ACS Nano 6, 10090-10097 (2012).

16. Menard, L. W., Mair, C. E., Woodson, M. E., Alarie, J. P. \& Ramsey, J. M. A device for performing lateral conductance measurements on individual double-stranded DNA molecule. ACS Nano 6, 9087-9094 (2012).

17. Soni, G. V. et al. A. Synchronous optical and electrical detection of biomolecules traversing through solid-state nanopores. Rev. Sci. Instrum. 81, 014301 (2010).

18. Squires, T. M. \& Quake, S. R. Microfluidics: Fluid physics at the nanoliter scale. Rev. Mod. Phys. 77, 977-1025 (2005)

19. Schoch, R. B., Han, J. \& Renaud, P. Transport phenomena in nanofluidics. Rev. Mod. Phys. 80, 839-883 (2008).

20. Dorfman, K. D. DNA electrophoresis in microfabricated devices. Rev. Mod. Phys. 82, 2903-2947 (2010).

21. Yasui, T. et al. DNA separation in nanowall array chips. Anal. Chem. 83, 6635-6640 (2011).
22. Bacri, L. et al. Dynamics of colloids in single solid-state nanopores. J. Phys. Chem. B 115, 2890-2898 (2011)

23. Tsutsui, M. et al. Single-nanoparticle detection using a low-aspect-ratio pore. ACS Nano 6, 3499-3505 (2012).

24. Fraikin, J.-L., Teesalu, T., McKenney, C. M., Ruoslahti, E. \& Cleland, A. N. A highthroughput label-free nanoparticle analyser. Nat. Nanotechnol. 6, 308-313 (2011).

25. Zhe, J., Jagtiani, A., Dutta, P., Hu, J. \& Carletta, J. A micromachined high throughput Coulter counter for bioparticle detection and counting. J. Micromech. Microeng. 17, 304 (2007)

26. Wu, X., Chon, C. H., Wang, Y., Kang, Y. \& Li, D. Simultaneous particle counting and detecting on a chip. Lab Chip 8, 1943-1949 (2008).

27. Pevarnik, M. et al. Polystyrene particles reveal pore substructure as they translocate. ACS Nano 6, 7295-7302 (2012).

28. DeBlois, R. W. \& Bean, C. P. Electrokinetic measurements with submicron particles and pores by the resistive pulse technique. J. Coll. Interf. Sci. 61, 323-335 (1977).

29. van Dorp, S., Keyser, U. F., Dekker, N. H., Dekker, C. \& Lemay, S. G. Origin of the electrophoretic force on DNA in solid-state nanopores. Nat. Phys. 5, 347-351 (2009).

30. Saleh, Q. A. Correcting off-axis effects in an on-chip resistive pulse analyser. Rev. Sci. Instrum. 73, 4396-4398 (2002).

31. Liu, H., Qian, S. \& Bau, H. H. The effect of translocating cylindrical particles on the ionic current through a nanopore. Biophys. J. 92, 1164-1177 (2007).

32. Davenport, M. et al. The role of pore geometry in single nanoparticle detection. ACS Nano 6, 8366-8380 (2012).

33. Liang, X. \& Chou, S. Y. Nanogap detector inside nanofluidic channel for real-time label-free DNA analysis. Nano Lett. 8, 1472-1476 (2008).

34. Tsutsui, M. et al. Single-molecule sensing electrode embedded in-plane nanopore. Sci. Rep. 1, 46 (2011).

\section{Acknowledgements}

A part of this work was supported by "Nanotechnology Platform Project (Nanotechnology Open Facilities in Osaka University)" of Ministry of Education, Culture, Sports, Science and Technology, Japan [No: F-12-OS-0016], by the Japan Society for the Promotion of Science (JSPS) KAKENHI Grant Number 24681032, and through its Funding Program for World-Leading Innovative R\&D on Science and Technology.

\section{Author contributions}

M. Tsutsui, T. K. and M. Taniguchi planned and designed experiments. N. Y. and S. T. fabricated microchannel samples. N. Y., M. Tsutsui and S. T. exhibited ionic current measurements and fluorescence observations. N. Y., M. Tsutsui, Y. H., H. S. and S. K. performed data analyses. M. Tsutsui, T. K. and M. Taniguchi co-wrote paper.

\section{Additional information}

Supplementary information accompanies this paper at http://www.nature.com/ scientificreports

Competing financial interests: The authors declare no competing financial interests.

License: This work is licensed under a Creative Commons

Attribution-NonCommercial-ShareAlike 3.0 Unported License. To view a copy of this

license, visit http://creativecommons.org/licenses/by-nc-sa/3.0/

How to cite this article: Yukimoto, N. et al. Tracking single-particle dynamics via combined optical and electrical sensing. Sci. Rep. 3, 1855; DOI:10.1038/srep01855 (2013). 